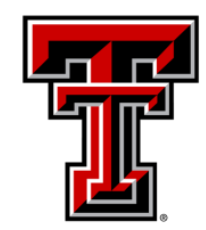

TEXAS TECH UNIVERSITY

Libraries"

\title{
COMPARATIVE EVALUATION OF TRANSMEMBRANE ION TRANSPORT DUE TO MONOPOLAR AND BIPOLAR NANOSECOND, HIGH-INTENSITY ELECTROPORATION PULSES BASED ON FULL THREE-DIMENSIONAL ANALYSES
}

\section{The Texas Tech community has made this publication openly available. Please share how this access benefits you. Your story matters to us.}

\begin{tabular}{|c|c|}
\hline Citation & $\begin{array}{l}\text { Q. Hu and R. P. Joshi, "Comparative evaluation of transmembrane } \\
\text { ion transport due to monopolar and bipolar nanosecond, high- } \\
\text { intensity electroporation pulses based on full three-dimensional } \\
\text { analyses," Journ. Appl. Phys. 122, } 034701 \text { (2017). } \\
\text { https://doi.org/10.1063/1.4994310 }\end{array}$ \\
\hline Citable Link & https://hdl.handle.net/2346/86912 \\
\hline Terms of Use & $\begin{array}{l}\text { This article may be downloaded for personal use only. Any other } \\
\text { use requires prior permission of the author and AIP Publishing. }\end{array}$ \\
\hline
\end{tabular}




\section{Comparative evaluation of transmembrane}

ion transport due to monopolar and bipolar nanosecond, high-intensity electroporation pulses based on full three-dimensional analyses

Cite as: J. Appl. Phys. 122, 034701 (2017); https://doi.org/10.1063/1.4994310

Submitted: 16 March 2017 . Accepted: 05 July 2017 . Published Online: 19 July 2017

Q. Hu, and R. P. Joshi
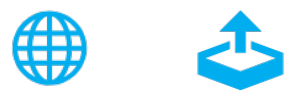

\section{ARTICLES YOU MAY BE INTERESTED IN}

Tutorial: Electroporation of cells in complex materials and tissue

Journal of Applied Physics 119, 201101 (2016); https://doi.org/10.1063/1.4949264

Assessing the electro-deformation and electro-poration of biological cells using a threedimensional finite element model

Applied Physics Letters 114, 063701 (2019); https://doi.org/10.1063/1.5079292

Numerical evaluations of membrane poration by shockwave induced multiple nanobubble collapse in presence of electric fields for transport through cells

AIP Advances 9, 045006 (2019); https://doi.org/10.1063/1.5085677

Challenge us. What are your needs for periodic signal detection?

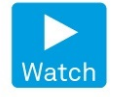

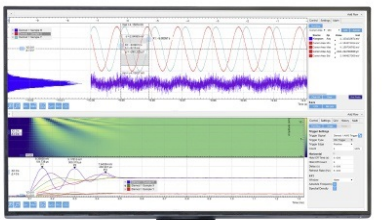

-
Zurich Instruments 


\title{
Comparative evaluation of transmembrane ion transport due to monopolar and bipolar nanosecond, high-intensity electroporation pulses based on full three-dimensional analyses
}

\author{
Q. $\mathrm{Hu}^{1}$ and R. P. Joshi ${ }^{2, \text { a) }}$ \\ ${ }^{1}$ School of Engineering and Technology, Central Michigan University, Mt. Pleasant, Michigan 48859, USA \\ ${ }^{2}$ Department of Electrical and Computer Engineering, Texas Tech University, Lubbock, Texas 79409, USA
}

(Received 16 March 2017; accepted 5 July 2017; published online 19 July 2017)

\begin{abstract}
Electric pulse driven membrane poration finds applications in the fields of biomedical engineering and drug/gene delivery. Here we focus on nanosecond, high-intensity electroporation and probe the role of pulse shape (e.g., monopolar-vs-bipolar), multiple electrode scenarios, and serial-versus-simultaneous pulsing, based on a three-dimensional time-dependent continuum model in a systematic fashion. Our results indicate that monopolar pulsing always leads to higher and stronger cellular uptake. This prediction is in agreement with experimental reports and observations. It is also demonstrated that multipronged electrode configurations influence and increase the degree of cellular uptake. Published by AIP Publishing. [http://dx.doi.org/10.1063/1.4994310]
\end{abstract}

\section{INTRODUCTION}

Applications of electric pulses to create nanopores in biological cell membranes can lead to orders-of-magnitude increase in plasma membrane permeability. ${ }^{1}$ This phenomenon, termed electroporation, depends upon the applied pulse duration and electric field strength. ${ }^{2-5}$ Traditionally, values of the pulse width ranged from tens of milliseconds to a few microseconds, with electric field intensities from $100 \mathrm{~V} / \mathrm{cm}$ to several kilovolts per centimeter. More recent developments in this arena encompass biomedical engineering, drug/ gene delivery, ${ }^{6-9}$ membrane poration (both reversible and irreversible), and controlled changes in cellular responses leading to immuno-therapy. ${ }^{10}$ Many of these recent applications involve the use of high intensity $(\sim 50-100 \mathrm{kV} / \mathrm{cm})$, nanosecond duration pulsed electric fields. This modality has also been shown to be useful ${ }^{11}$ including applications such as electrically triggered intra-cellular calcium release, ${ }^{12,13}$ shrinkage of tumors, ${ }^{14,15}$ temporary blockage of action potential in nerves, ${ }^{16}$ and activation of platelets for accelerated wound healing. ${ }^{17}$ In addition, the use of high-intensity electric pulses in the microsecond regime is emerging as a relatively safe, effective and minimally invasive ablation modality for treating tumors. ${ }^{18}$ The non-thermal nature of this excitation (unlike the heating caused by microwave or radiofrequency ablation) allows for treatment even in close proximity to critical structures and/or large vessels. ${ }^{19}$ Another important application of electroporation is in electrochemotherapy $\mathrm{y}^{20,21}$ and these recent reviews provide a thorough meta-analysis and discussion on this issue and other biological effects of nanosecond electroporation.

From the standpoint of drug/gene delivery, besides the biologically related variables, ${ }^{21-24}$ other important factors are the electrode shape and geometries, as well as the electrical pulsing parameters. ${ }^{25,26}$ Membrane electropermeabilization begins when the applied field exceeds a critical

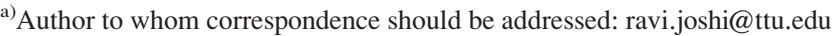

transmembrane potential (TMP) of about $1 \mathrm{~V}$ (Ref. 27) and is followed by electrophoresis-based molecular uptake. ${ }^{28-30}$ Thus, both permeabilization (which opens the cell to transport processes) and subsequent delivery are controlled in large part by parameters such as the field strength, pulse duration, number of pulses, and pulse shape. Multi-electrode systems have also been used (e.g., two parallel plates or six electrode arrays) for enhancing the delivery. ${ }^{31,32}$

The role of the electrical pulse shape on biological cell membrane permeabilization has been the topic of multiple studies, ${ }^{33-37}$ though most have focused on millisecond pulses. Most of these previous studies concluded that bipolar pulses appear to have a stronger effect on electropermeabilization as compared to monopolar pulses. The reason given was that bipolar pulses compensate for the asymmetry inherent in the permeabilized areas at the poles of the cell that is introduced by the resting transmembrane voltage. However, more recent studies with much shorter pulses (300 ns and $600 \mathrm{~ns}$ ), applied to Chinese Hamster Ovary cells showed bipolar pulses to be less effective at electropermeabilization. ${ }^{38,39}$ They were also found not to be as efficient in increasing the intracellular calcium concentration. Now, application of pulses in this nanosecond domain is certainly important for a variety of reasons. For example, the short duration pulses mitigate muscle contractions, and so are a useful modality for the ablative treatment of solid tumors. ${ }^{40,41}$ Eliminating contractions helps improve the procedural safety of patients, since the need for neuroparalytic drugs to inhibit muscle contraction is then virtually eliminated. The short duration also eliminates localized thermal heating and reduces the potential for side-effects such as hyperthermia or cell damage.

Here, our primary focus is on transmembrane transport and cellular inflows driven by external electric pulses with the shorter nanosecond duration pulses. The molecular transport following membrane electroporation is studied through a full three-dimensional (3D), time-dependent continuum model. Almost all previous studies have relied on simpler twodimensional treatments by invoking azimuthal symmetries of 
spherical cells. For example, electric fields for different electrode configurations were estimated either numerically based on finite element or finite difference methods, ${ }^{42-45}$ or used analytical solutions. ${ }^{46}$ However, for cellular intake in the presence of an external electric field following poration, such symmetries can only be invoked at the very beginning. The very process of inflows through the cell membrane in response to any practical electrode arrangement (such as the two-plate or six-needle arrays, which do not have a three-dimensional symmetry) invalidates the simple approximation. Hence, a full 3D analysis is really required. An additional benefit of a full three-dimensional treatment would be its applicability towards non-spherical cells.

In this contribution, the role of pulse width on cellular inflows for both monopolar and bipolar stimulation is evaluated. In addition, the response to voltages applied at multiple electrodes is also probed. From the standpoint of a practical application, the pulse width and pulsing sequence for multiple electrodes would influence the uniformity and degree of cellular uptake. The multi-pronged electrode configurations offer spatial flexibility, and phased array-like behavior could be achieved through judicious pulse sequencing. The present simulations also include comparisons between monopolar and bipolar pulse responses.

\section{ELECTROPORATION MODELING DETAILS}

\section{A. Transmembrane potential}

An approach to calculating the transmembrane potential is through a time-domain nodal analysis involving a distributed equivalent circuit representation of a cell and its membrane structures. The dynamic electric field in the simulation region can then be obtained from the node voltages. Details of this method and its implementation have been given elsewhere ${ }^{47,48}$ and hence, only a brief outline will be discussed here. Essentially, the entire cell volume was broken up into finite segments, and each segment was represented by a parallel resistive-capacitive $(R C)$ combination to account for the current flow and charging effects. The computational region was a sphere that included the cell with its outer membrane, the aqueous intra-cellular medium, and the surrounding suspension, discretized along the $r, \theta$, and $\varphi$ directions. For simplicity, the cell membrane itself was taken as an integral unit, i.e., this subregion was not further discretized. For interior nodes, the current continuity equation is of the form

$$
\sum_{k=1}^{6}\left(\sigma E+\varepsilon \frac{\partial E}{\partial t}\right)_{k} \times A_{k}=\sum_{k=1}^{6} I_{k}=0,
$$

where $I_{k}$ are currents along the six faces of an elemental cube with surface areas $A_{k}$, the local electric field is $E$, while $\varepsilon$ and $\sigma$ are the average permittivity and conductivity at the site of each cube. A schematic of the discretized geometry is shown in Fig. 1(a). Details of the equations for each elemental volume shown in Fig. 1(a), can be found in the Appendix. Combining the current continuity then leads to $N$ equations for the $N$ unknown node voltages
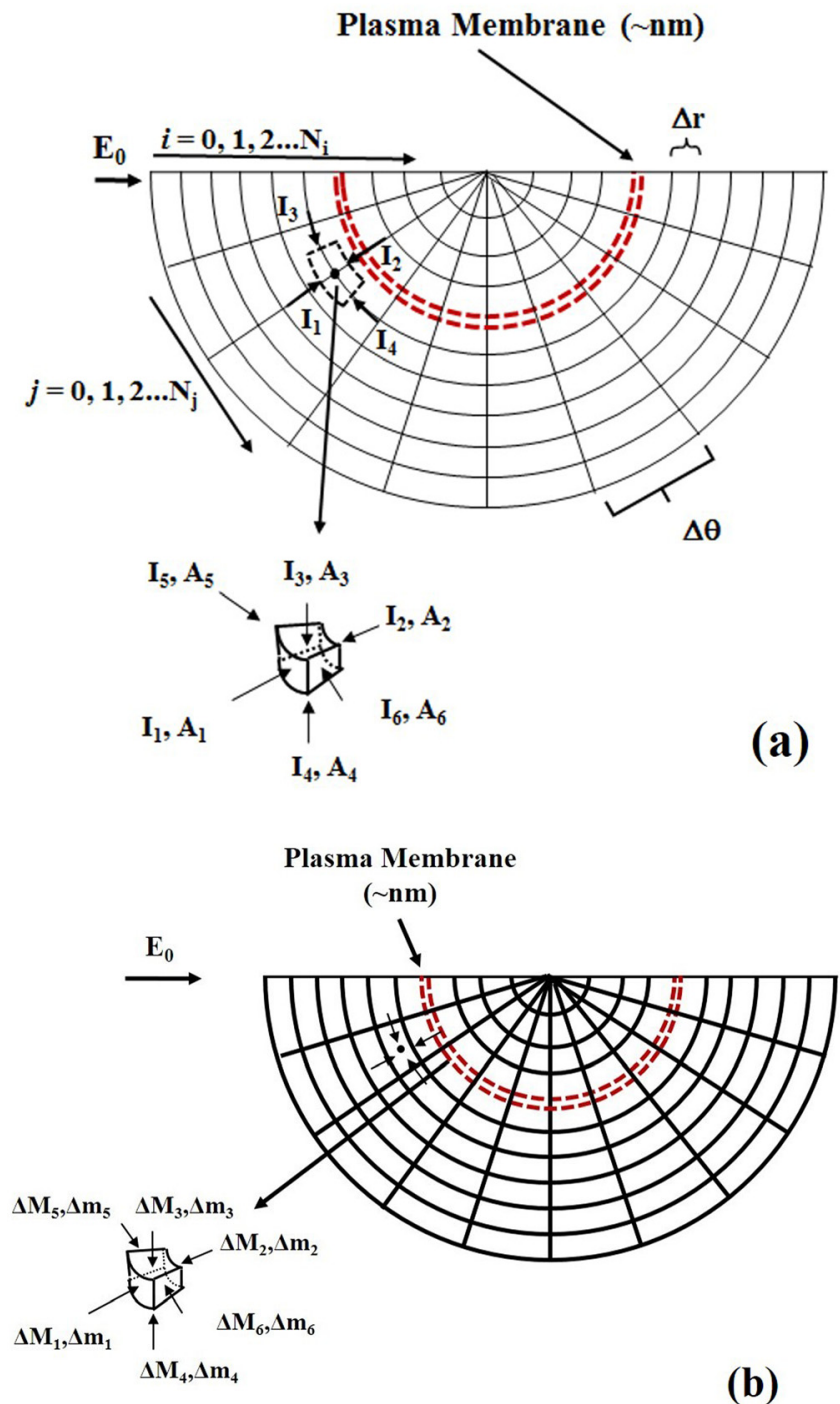

FIG. 1. Sketch of the discretization used for an elemental volume encompassing each grid point with six surfaces in: (a) transmembrane potential calculations and (b) ion concentration calculations.

which can be solved using matrix de-composition techniques. Potentials on each node were updated at every time step based on the boundary conditions imposed by the externally applied field, and the dynamic membrane conductivity which changes over time due to electroporation. Nodes on the periphery of the simulation region of radius $R$, were assigned potential values as boundary conditions in keeping with the external electric fields. For example, for an external field of magnitude $E_{0}$ directed along the $z$ direction, the boundary nodes were set to values of $-E_{0} R$ $\cos (\theta)$, with $\mathrm{R}$ being the angular location of the node relative to the $z$-axis. For fields applied along two or more principal, appropriate superposition of voltages were applied as the potential at the boundary nodes.

\section{B. Membrane nanopores and ionic flow}

Pore generation, growth, and size-evolution required to characterize time-dependent membrane conductivity were obtained based on the Smoluchowski continuum theory. ${ }^{49-53}$ 
Here the full Smoluchowski model was solved, rather than using the asymptotic approximation of Neu and Krassowska. ${ }^{49}$ This enabled calculations of the time-dependent pore density $N(t)$ at different locations on the membrane surface. ${ }^{54}$ Table I lists the values used in the present simulation work. Application of the external electric field drives the pore generation and growth, through which ionic flow can occur. The current density $J_{E P}(t)$ through a membrane pore is given as: $J_{E P}(t)=i_{E P}(t) N(t)$, where $i_{E P}(t)$ is the current through a pore and $N(t)$ is the pore density. The pore areas on the membrane at different mesh locations and times, as well as the electric field $E$ can be obtained, which enables computations of ion flow across the cell membrane. Diffusive flow of ions through membrane pores was modeled as ${ }^{30}$

$$
J_{\text {diff }}=-D * H * K * \frac{\partial C_{i, j}}{\partial x} .
$$

In the above, $J_{\text {diff }}$ is the flow rate per area, $H$ denotes the Hindrance factor which is related to average pore sizes with $H \sim 1$ (Ref. 55) being used for pore sizes larger than ions, $K$ is the partition factor $\left(=0.83\right.$ for $\left.\mathrm{Ca}^{+2}\right), D$ is the diffusion coefficient, and $C_{i j}$ is the concentration of the $i$ th ion at grid point $j$. The number of ions going through the membrane in unit time was calculated by: $\frac{\Delta m_{1}}{\Delta t}=J_{\text {diff }} * A_{\text {pore }}$, with $A_{\text {pore }}$ being the cumulative sum of the pore areas. For other layers not adjacent to the cell membrane, $J_{\text {diff }}=-D \frac{\partial C}{\partial x}$ was used. The drift pore currents $i_{E P}(t)$ and changes in ionic concentration $\Delta C$ were taken as

$$
\Delta C_{i, j}=\sum_{i=1}^{6} \Delta M_{i} / V_{s}
$$

In the above expression

$$
\frac{\Delta M_{1}}{\Delta t}=\left\{\begin{array}{l}
C_{i-1, j, k} V_{s} E_{1} \mu A_{\text {pores }}, E_{1}>0 \\
C_{i, j, k} V_{s} E_{1} \mu A_{\text {pores }}, E_{1} \leq 0
\end{array}\right.
$$

where $C_{i, j, k}$ is the molarity of the unit volume inside cell, $V_{s}$ is the space volume of the unit, $\mu$ is the ion mobility which equals $\frac{D q Z}{K_{b} T}$, with $D$ being the ion diffusion coefficient ( $\left.\sim 0.8 \times 10^{-5} \mathrm{~cm}^{2} / \mathrm{s}\right), k_{B}$ is the Boltzmann constant, $T$ is the temperature in Kelvin, $q$ is the electronic charge, $z$ is the valence charge of the ions (e.g., $z=2$ for calcium ions), and

TABLE I. Simulation parameters used.

\begin{tabular}{lc}
\hline \hline Pulse types & Monopolar and bipolar \\
\hline Pulse rise time & $1.5 \mathrm{~ns}$ \\
Pulse fall time & $1.5 \mathrm{~ns}$ \\
Membrane permittivity & $4 \times 8.85 \times 10^{-12} \mathrm{~F} / \mathrm{m}$ \\
Membrane conductivity & $5.3 \times 10^{-6} \mathrm{~S} / \mathrm{m}$ \\
Membrane thickness & $5 \mathrm{~nm}$ \\
Cell radius & $0.25 \mu \mathrm{m}$ \\
Ca ion diffusion coefficient & $0.8 \times 10^{-5} \mathrm{~cm}^{2} / \mathrm{s}$ \\
Grid points R-direction & 31 grid points \\
Grid points $\Theta$-direction $(0-\pi / 2)$ & 11 grid points \\
Grid points $\Phi$-direction $(0-2 \pi)$ & 20 grid points \\
\hline \hline
\end{tabular}

$E_{1}$ is the average electrical field across surface $A_{1}$, as shown in Fig. 1(b). From Eq. (4), one can obtain the moles of calcium ions going into the cell through the pores for $E_{1}>0$, or getting out of the cell pores for $E_{1} \leq 0$. Thus, the pore current $i_{E P}(t)$ in a time interval $\Delta t$ is given as: $=\Delta M_{1}$ $\Delta t z N_{A}$, with $N_{A}$ being the Avogadro number. For simplicity, only calcium ion transport was simulated, though the model and technique is general, and can be used for the analyses of multi-ion membrane flows. However, to maintain charge neutrality, an equal concentration of immovable chlorine ions was assumed in the simulation space outside the cell.

\section{SIMULATION RESULTS AND DISCUSSION}

\section{A. Transport due to monopolar and bipolar pulses}

The above model was used to study ion flows into cells through porated membranes in response to external electric field pulsing. Table I lists the various parameters used. These are standard values that have typically been reported in the literature. For concreteness, the transport of calcium ions was simulated with a diffusion coefficient of $0.8 \times 10^{-5}$ $\mathrm{cm}^{2} / \mathrm{s}$. For the ion concentration, a density of $2 \mathrm{mM}$ was initially set at the start in the aqueous medium outside the cell, while the initial values within each elemental the cell volume were set to zero. A representative schematic of bipolar and monopolar pulses with a near-rectangular shape used in the present simulations is shown in Fig. 2. The separation time between the end of any pulse and the beginning of the next pulse is denoted by $T_{s}$. Each of the repetitive cycles (varied for different simulations) was chosen to have short rise- and fall-times of $1.5 \mathrm{~ns}$. The externally applied electric field magnitudes were varied, though for concreteness, a value of $100 \mathrm{kV} / \mathrm{cm}$ has been shown in Fig. 2. Monopolar pulses are similar to the bipolar train, except that the negative fields are reversed to yield positive values at all times. For simplicity, only calcium ions were assumed to be present in the

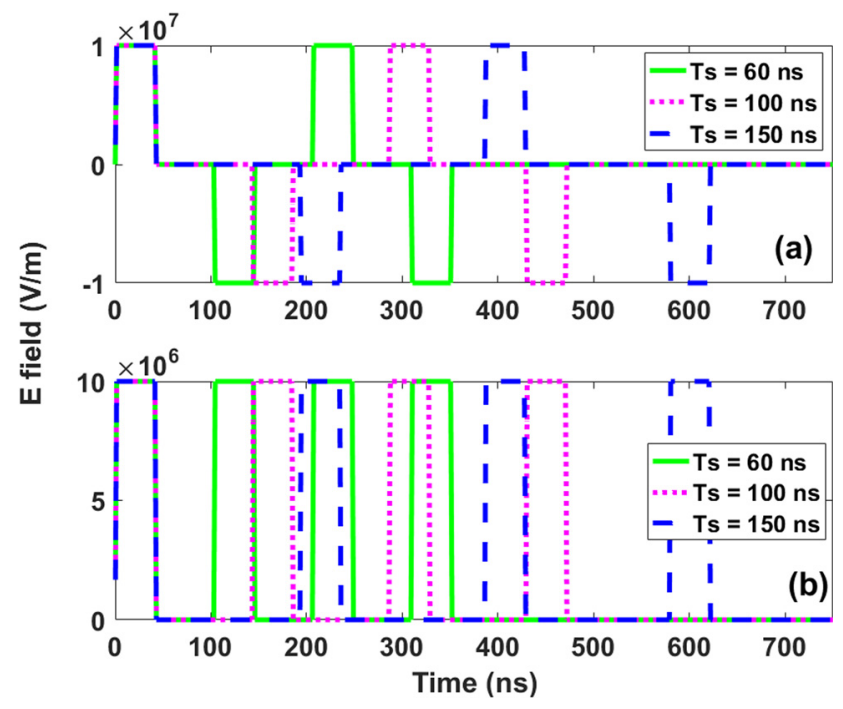

FIG. 2. A representative time-dependent bipolar and monopolar waveforms used for the simulations. The separation time between the end of any pulse and the beginning of the next pulse is denoted by $T_{s}$. 
extracellular medium though the approach is general and multi-ion situations can easily be treated.

Simulations of the time dependent calcium concentration within the cell in response to both bipolar and monopolar pulses of the type shown in Fig. 2 were carried out. These results are shown in Fig. 3, with a pulse "ON" time of $60 \mathrm{~ns}$, a peak field of $82 \mathrm{kV} / \mathrm{cm}$, and an "OFF"-time of $60 \mathrm{~ns}$. The pulses were applied at the 9- and 3-o'clock positions, and hence, assumed to be along only one of the three orthogonal axes. The monopolar pulses had the same pulse parameters as the bipolar train except that all the pulses were positive in sign. For comparison, results with and without the inclusion of diffusive flows are also given. Figure 3 shows an initial delay for calcium ion entry since a finite time is required for membrane electroporation, which is then followed by ion inflows into the cell. The inflow due to drift is predicted to be rapid beyond the first pulse for the monopolar case, and so an upsurge is seen after about $121 \mathrm{~ns}$. For the bipolar pulses, on the other hand, though pore formation is predicted to occur, the movement of ions keeps reversing in the direction.

The difference between the two cases is perhaps best understood in terms of the transmembrane potentials (TMPs) over time, since the field values drive the flow. The TMP values are shown in Figs. 4(a) and 4(b) at the 9-o'clock position for the two polarities. With monopolar pulses, the TMP rises four times corresponding to the start of the four pulses. The highest TMP (and hence transmembrane electric field) value is reached for the first pulse. At subsequent times, due to pore formation, the membrane conductivity increases leading to lower voltage drops and reduced TMP values. In this monopolar pulse scenario, the positive external electric field direction is from left-to-right (i.e., from the 9- to o3o'clock positions). Hence, positive ions go into the cell at the 9 o'clock position upon pore formation; but there is no ionic flow at the opposite end for the 3 o'clock position because the cell interior is devoid of any ions. The ionic throughput for this case continues from the left side and is most strong around the neighborhood of the 9-o'clock region. For bipolar pulses, the initial field direction is from left-to-right for the

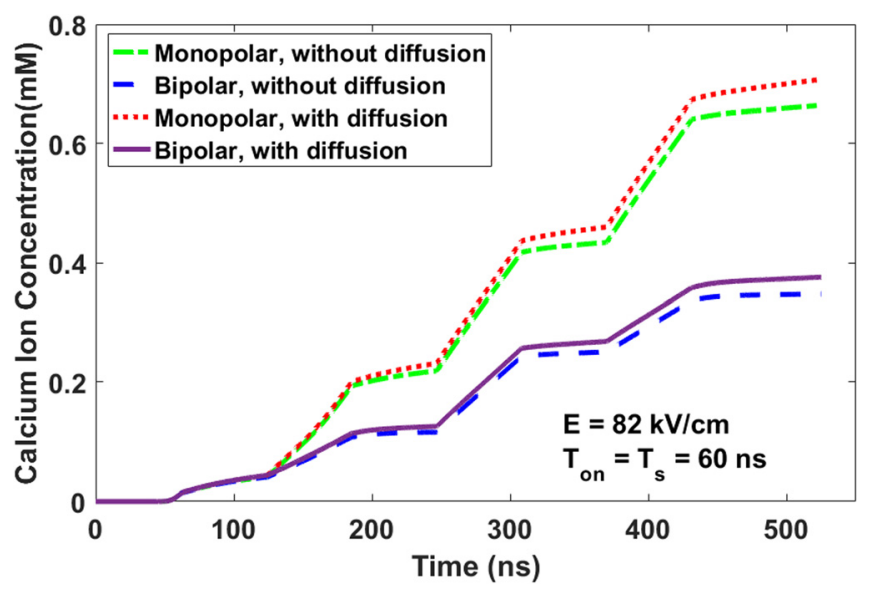

FIG. 3. Simulation results showing the temporal growth of the calcium concentration inside a cell subjected to bipolar and monopolar pulses, with and without diffusion taken into account.
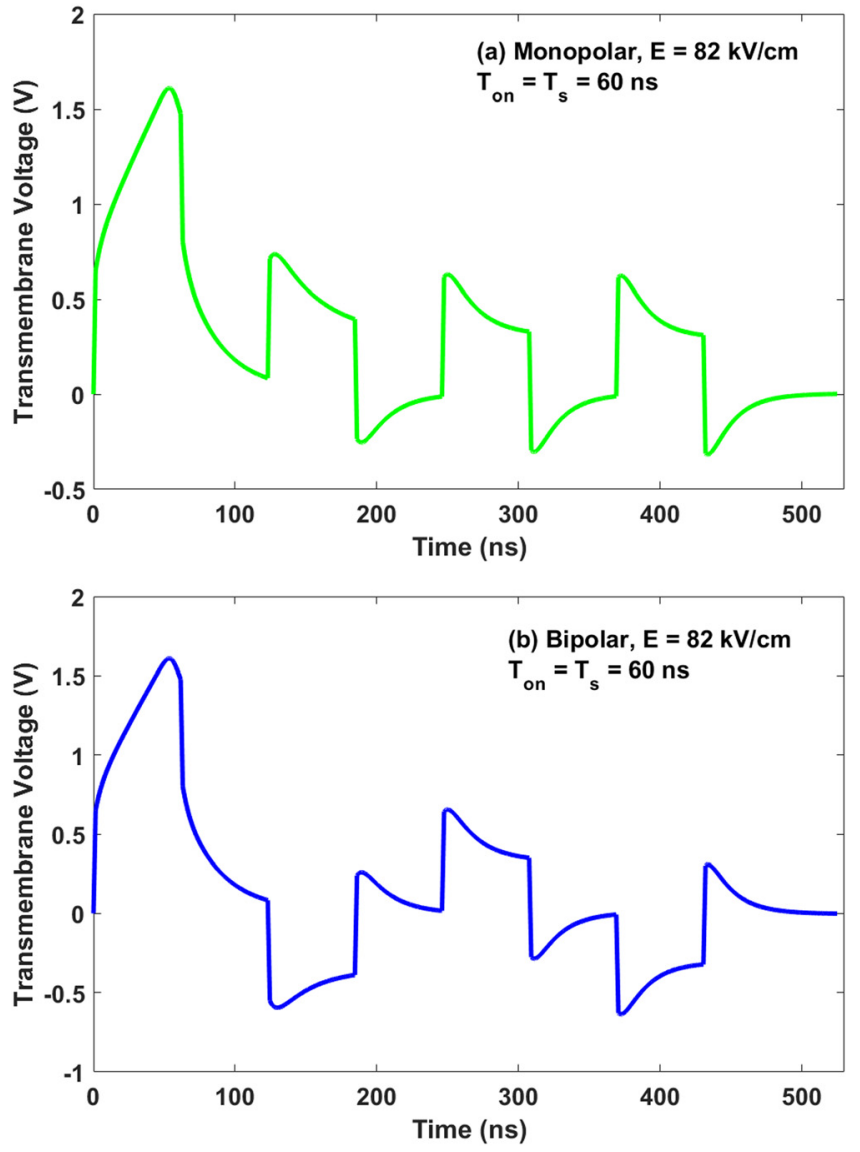

FIG. 4. Transmembrane potentials at the 9-o'clock position for the two pulsing cases. (a) Monopolar pulses and (b) bipolar pulses.

first pulse, leading to a positive TMP at the 9-o'clock position. However, this leads to a depletion of ions in the aqueous medium outside (but close to) the cell surface in the neighborhood of the 3-o'clock position. Since diffusion is a slow process, the recovery through ion flowback is slow, and hence the neighborhood around 3-o'clock remains relatively depleted. The second pulse, of reversed polarity, creates a negative TMP at the 9-o'clock location [as seen in Fig. 4(b)] with the field direction pointing from right-to-left. At this point in time, ion inflow into the cell is from the 3-o'clock neighborhood. Given the depletion of ions in the aqueous medium in this region though, the inflow is not as strong. Furthermore, reversal in the electric field leads to some outflow of ions from the pores previously formed at the 9o'clock neighborhood. The net effect is that the ion content inside the cell is comparatively much lower than that for the monopolar case. So overall, the two main points to note from Fig. 3 are that: (i) with diffusion taken into account, the concentration in the cell is slightly higher, though the difference is not significant. (ii) The response in terms of ion inflow from monopolar pulsing is much stronger than that for bipolar pulses.

Next, simulations were carried out to gauge the amount of calcium inflows for two pulses having the same energy content but different electric field amplitudes and pulse durations. A simple calculation based on electrophoretic flow indicates that the flow volume $F_{\text {in }}$ should roughly be 


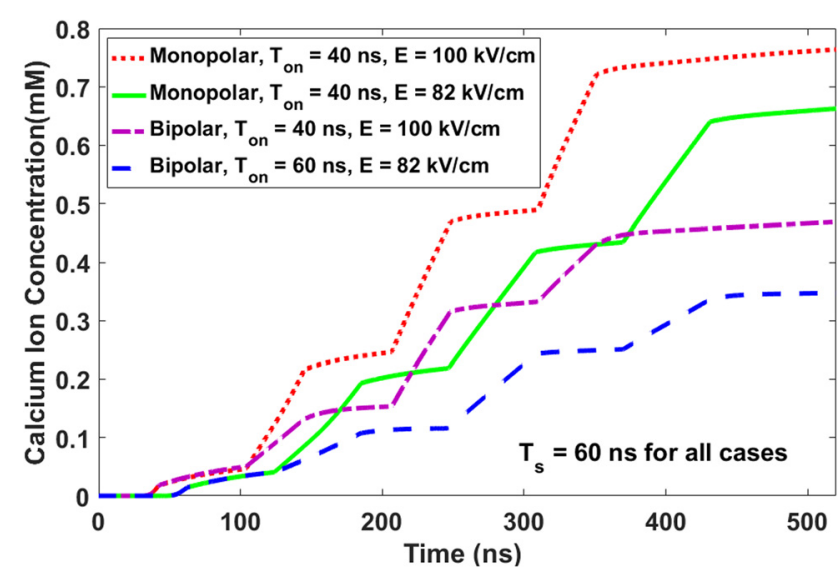

FIG. 5. Temporal growth of the calcium uptake for pulses of equal energy. Pulse ON-times of $40 \mathrm{~ns}$ and $60 \mathrm{~ns}$ were used, with corresponding field strengths of $100 \mathrm{kV} / \mathrm{cm}$ and $82 \mathrm{kV} / \mathrm{cm}$, respectively. The results include both bipolar and monopolar pulsing scenarios.

dependent as: $v A d t$, where $v$ is the drift velocity, $d t$ is the time duration, and $A$ is the effective pore area. The flow $F_{i n}$ would then scale as $F_{\text {in }} \sim E A d t$ at constant mobility. The energy $G$, on the other hand, scales as: $G \sim E^{2} d t$. Hence, for two pulses having the same energy but different electric fields $\left(E_{1}, E_{2}\right)$ and durations $\left(d t_{1}, d t_{2}\right)$, one gets: $\left[F_{i n 1} / F_{i n 2}\right] \sim$ $\left(A_{1} / A_{2}\right)\left(d t_{1} / d t_{2}\right)^{1 / 2}$. The simulation results for the timedependent inflow are shown in Fig. 5 for pulse ON-times of $40 \mathrm{~ns}$ and $60 \mathrm{~ns}$, having peak field amplitudes of $100 \mathrm{kV} / \mathrm{cm}$ and $82 \mathrm{kV} / \mathrm{cm}$, respectively. The curves reveal that the shorter pulse (having the higher electric field of $100 \mathrm{kV} / \mathrm{cm}$ ) drives more ions into the cell, as compared to a longer pulse of the same energy. This indicates that pore areal changes after the initial pulse are consequential and field-dependent. The outcome is a result of a higher density of pores at the higher fields, despite the shorter time duration. This is also indicative of a short pore formation time at these high fields, in keeping with experimental observations. ${ }^{56}$ Also, as with the previous result of Fig. 3, the monopolar pulsing is more effective at cellular loading.

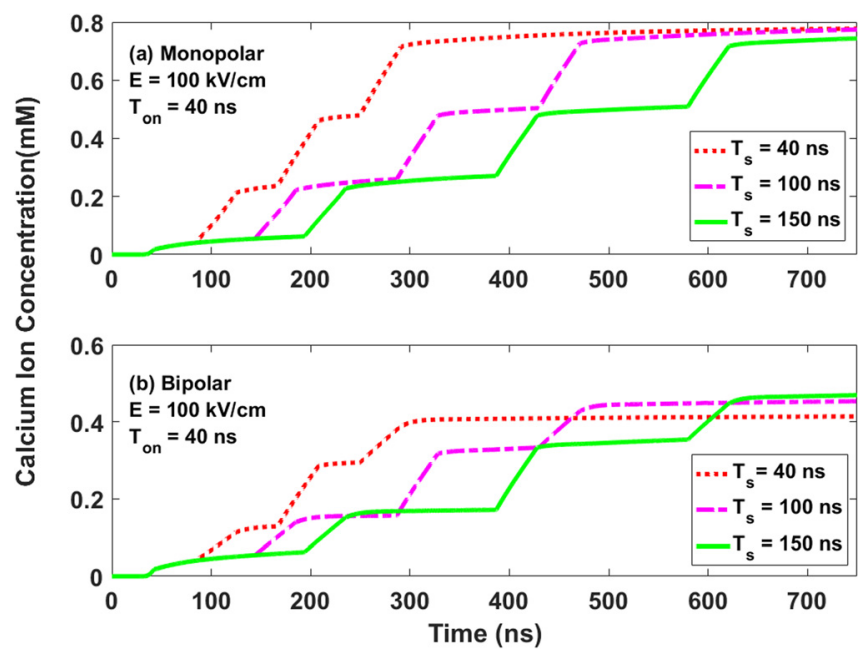

FIG. 6. Simulation results probing the effect of changes in the pulse separation times $T_{s}$. (a) Monopolar pulses and (b) bipolar pulses.
The role of pulse separation (i.e., the pulse OFF time which represents the time interval separating the active pulses) on calcium ion inflows was probed next. Figure 6 shows the simulation results for different pulse separation times $T_{s}$. A four-pulse monopolar sequence is shown in Fig. 6(a). The ON-time remained at $40 \mathrm{~ns}$, while the riseand fall-times were set at $1.5 \mathrm{~ns}$ in all cases. The peak electric field magnitude was taken to be $100 \mathrm{kV} / \mathrm{cm}$. As might be expected, the calcium concentration in the cell decreases as the pulse delay is increased. This occurs due to two reasons. First, the calcium concentrations that might have been built up at the cell membrane boundary during the ON-time, diffuse and slowly recover back to a more uniform, equilibrium value. As a result, a lower density of calcium is available in the vicinity of the cell surface for re-entry. Second, the slow but gradual pore closing during the OFF state, reduces the total area of ion inflow through the membrane surface.

With the use of bipolar pulses, the scenario is somewhat different as shown in Fig. 6(b) for the same geometry and pulse parameters. Well after the pulse train is over, higher inflows are predicted to be from pulses with the longer delay. This result can qualitatively be understood as arising from the recovery of the ion concentrations outside, but in the immediate vicinity, of the cell surface. As already mentioned, one phase of the pulse drives the calcium away from the cell surface and deeper into the aqueous medium at either the 9- or 3-o'clock (or any pair of diagonal) positions. Hence, when the polarity is reversed for the succeeding stimulation, the amount of calcium available for inflow is lower due to the depletion that has taken place during the preceding pulse. However, as the pulse delay time $T_{s}$ is increased, such depletions are quenched (and could nearly be restored) due to local diffusive flows. This increases the availability of calcium for cellular entry at the subsequent pulse. Expectedly though, the total magnitudes flowing into the cell are much lower for bipolar pulsing, as opposed to the previous monopolar case.

For completeness, simulations probing the effect of a small $5 \mathrm{~ns}$ pulse separation time $\left(T_{s}\right)$ for bipolar pulses were performed, with the results shown in Fig. 7 for two different
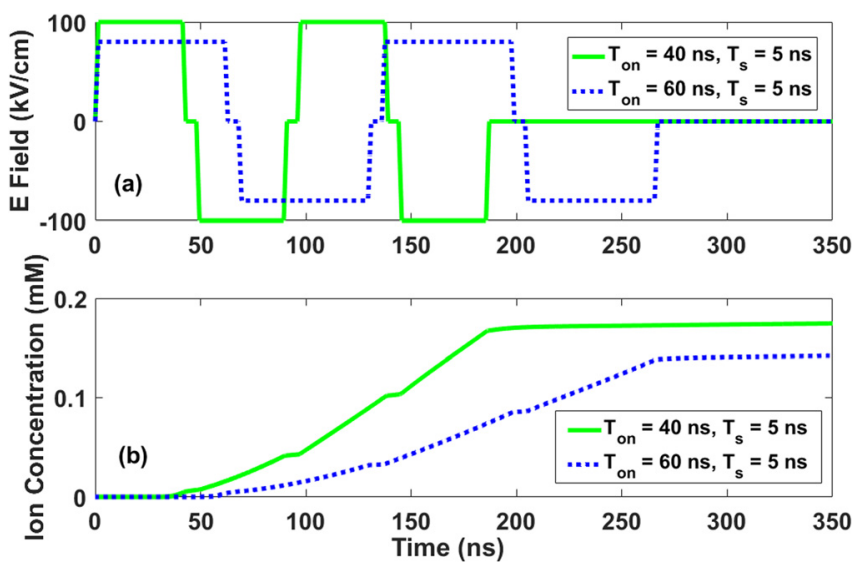

FIG. 7. Simulation results probing the effect of a small $5 \mathrm{~ns}$ pulse separation time $T_{s}$ for bipolar pulses. 
ON-times of $40 \mathrm{~ns}$ and $60 \mathrm{~ns}$. Based on equal energy, the electric field for the longer $60 \mathrm{~ns} \mathrm{ON}$ time pulses was lower. In keeping with the trends of Fig. 5, the cellular inflow for the lower electric field (i.e., the $60 \mathrm{~ns}$ ON time case) was also correspondingly lower. But more importantly, the uptake with the much shorter $5 \mathrm{~ns}$ pulse separation time, is predicted to be much less than the values with the longer separation. This is qualitatively in keeping with the observations of Ibey et al. $^{38}$ and Pakhomov et al. ${ }^{39}$ who reported practically no calcium uptake for such bipolar pulses. Though a small amount of ion inflow is predicted in Fig. 7, the value is small enough that it might not have been observed experimentally. Besides, the temporal resolution available in the experimental measurements was not on such a fine nanosecond scale, but rather was probed on a time scale of seconds after the onset of the pulse. The long time scales could have meant a much greater internal diffusion, and hence dilution, of any meagre intracellular flows that might have occurred during the bipolar pulsing.

Based on the above results, the following conclusions seem to emerge with regards to the mechanisms for the lower cellular uptake by bipolar pulses. In this context, Gianulis et al. ${ }^{57}$ discussed three potential mechanisms previously proposed for the reduced uptake: (i) the assisted membrane discharge which shortens the time when the membrane is above the critical breakdown potential, (ii) poration involves charge transfer and thus can be reverted by the electric field reversal, and (iii) the possibility to drive charged ions out of the cell upon electric field reversal. Our calculations predict the uptake, in the case of bipolar pulsing, to be smaller because high transmembrane potentials (TMPs) are created over a shorter time duration. For example, a comparison between Figs. 4(a) and 4(b) reveals the absolute values of the TMP over the 200 ns-to- $400 \mathrm{~ns}$ time span remain relatively lower for the bipolar case. The primary reason is that with unidirectional pulsing, a subsequent pulse can start to raise the TMP to a higher level (as compared to a bipolar pulse) if the starting value was not at zero. For a bipolar pulse, on the other hand, if one is at a nonzero TMP value just prior to a subsequent pulse, the magnitude first goes through zero before being enhanced in the opposite direction.

Furthermore, based on the present results, the Ca-ions driven into the cell through the membrane pores do not flow out immediately from the entry sites upon polarity reversal. Instead, ions continue to flow in from the membrane region located diametrically on the opposite side. The inflow though is less, since the external ion concentration on the diametrically opposite side has fallen due to the prior movement from the first pulse. Thus, charge reversal is not seen to play a dominant role in the present simulations.

\section{B. Multiple electrode pulsing}

Next for completeness, the possibility of applying the voltage pulse in a sequential versus simultaneous manner was compared. The sequential (or series) application represented a situation wherein the electric pulse was first applied at the 9- and 3-o'clock positions (i.e., the field was taken to be along the horizontal direction). Then after a $60 \mathrm{~ns}$ delay, the same pulse was applied in an orthogonal direction to pass through the 12- and 6-o'clock positions. With simultaneous application, on the other hand, both the 9- and 3-o'clock positions, as well as the 12- and 6-o'clock locations, were exposed to the external voltage pulse simultaneously. Four sets of pulses were simulated. Thus, for the simultaneous application four distinct pairwise events occurred, while in the sequential case, eight separate events took place.

The results obtained for the cellular uptake are shown in Fig. 8 for a peak field amplitude of $80 \mathrm{kV} / \mathrm{cm}$, a pulse ONtime of $40 \mathrm{~ns}$ with a OFF-time of $60 \mathrm{~ns}$. Only the monopolar pulses were chosen since this modality has been shown to invoke a larger cell uptake, and hence is of higher interest. In Fig. 8 , and the cellular uptake is predicted to be larger when both excitations are simultaneously applied at the 9-3 and $12-6$ o'clock positions. Four distinct jumps in the ion concentration over time can be seen in the plot corresponding to the four distinct events for simultaneous pulsing. On the other hand, with serial pulses, eight distinct jumps in the ion concentration over time are evident in Fig. 8 as expected. Synergies for the monopolar case become apparent, for example, from the ion concentration around $400 \mathrm{~ns}$. The predicted value of $\sim 0.6 \mathrm{mM}$ at this time for the simultaneous pulsing case is seen to be more than twice the concentration obtained for the serial case. Clearly, the two mutually orthogonal pulses affect a larger fraction of the cell surface, and hence create a much larger effective pore area, to enhance the ion throughput for simultaneous excitations. This outcome seems reasonable since for this situation, pores get formed at both pairs of polar caps. Furthermore, poration is enhanced due to additive contributions of the electric fields even over the off-axis regions.

Finally, as a test of the possible synergies, simulations with multiple pulses were performed. Since monopolar pulses have consistently been shown to achieve higher

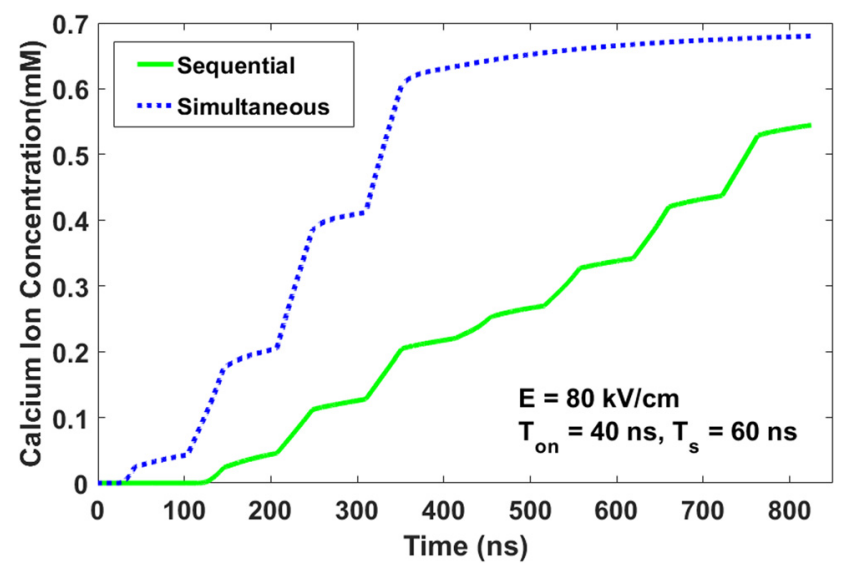

FIG. 8. Results of cellular uptake comparing sequential and simultaneous application of the external voltage pulse for monopolar pulses having a peak amplitude of $80 \mathrm{kV} / \mathrm{cm}$. Four sets of two orthogonal simultaneous pulses were applied for the simultaneous case. For the sequential case, a total of 8 separate pulses were applied, first along one direction and then along the orthogonal direction with a $60 \mathrm{~ns}$ delay. This delayed pair was repeated four times. 
cellular inflow, results from the bipolar pulse cases were omitted for brevity. Total ion concentration changes inside the cell were evaluated in response to monopolar pulses in the train having 40-ns duration, 1.5-ns rise-, 1.5-ns falltimes, and 60-ns off times. The electric field intensity was set at $80 \mathrm{kV} / \mathrm{cm}$. In order to keep the overall energy delivered to the system equal, three cases are studied: (i) a scenario with six pulses applied along the x-direction, (ii) a second situation with three pulses applied along both the y- and zdirections simultaneously, and (iii) the simultaneous application of two pulses along the $\mathrm{x}-, \mathrm{y}-$, and $\mathrm{z}$-directions for the greatest synergy. It may be emphasized, however, that there can be numerous ways of choosing values from a parameter space for comparisons. Here, we have opted to use one simple means, that of maintaining the input energy. Nonetheless, it must be clarified that electroporation is an especially complicated and sequential process, and there is no simple scaling formula that can factor in all the variables in a simple way.

In any event, the results shown in Fig. 9 for the above three simulation cases reveal that the largest throughput occurs when all three pulses are applied simultaneously along the three mutually orthogonal directions. The weakest response is when pulses are only applied along one direction, even though their numbers are higher. All this collectively points to the inherent synergies, and suggests that judicious pulse manipulation, sequencing, and multi-directional targeting can lead to enhanced cellular delivery. Though the threedimensional simulations were carried out for spherical cells, it seems intuitive that such multi-directional pulsing would be even more advantageous for cells or tissues having irregular shape. By hitting the collective mass or various irregularly shaped cells at multiple sites, a stronger and more prolific bio-response can be expected. This is quite likely in the case of tumor tissues. Ideas associated with phased array delivery could also be probed as the next logical step in this direction.

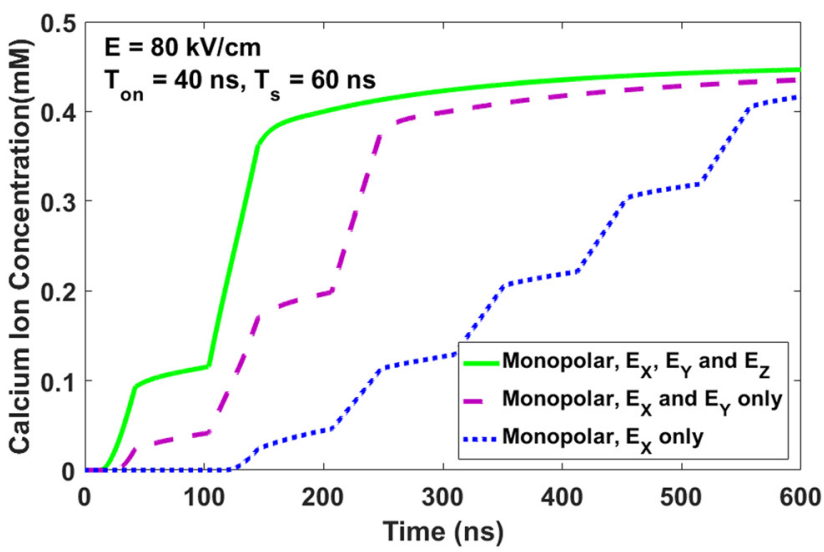

FIG. 9. Evolution of the ion concentration inside the cell in response to pulses in a train with 40-ns durations, $1.5 \mathrm{~ns}$ rise- and fall-times, and 60-ns off times. The peak field amplitude was chosen to be $80 \mathrm{kV} / \mathrm{cm}$. The three curves shown correspond to six pulses applied along the $\mathrm{x}$-direction, three pulses applied along both the $\mathrm{x}$ - and $\mathrm{y}$-directions simultaneously, and two pulses applied along the $\mathrm{x}-, \mathrm{y}-$, and $\mathrm{z}$-directions simultaneously. The energy delivered to the system for all three cases was equal.
The strong performance of simultaneous pulsing in Fig. 9 is made clearer through the time-development of transmembrane potentials (TMPs) across various points on the membrane surface. The simulation results obtained are shown in Fig. 10 at the 30 ns time instant for the three cases.
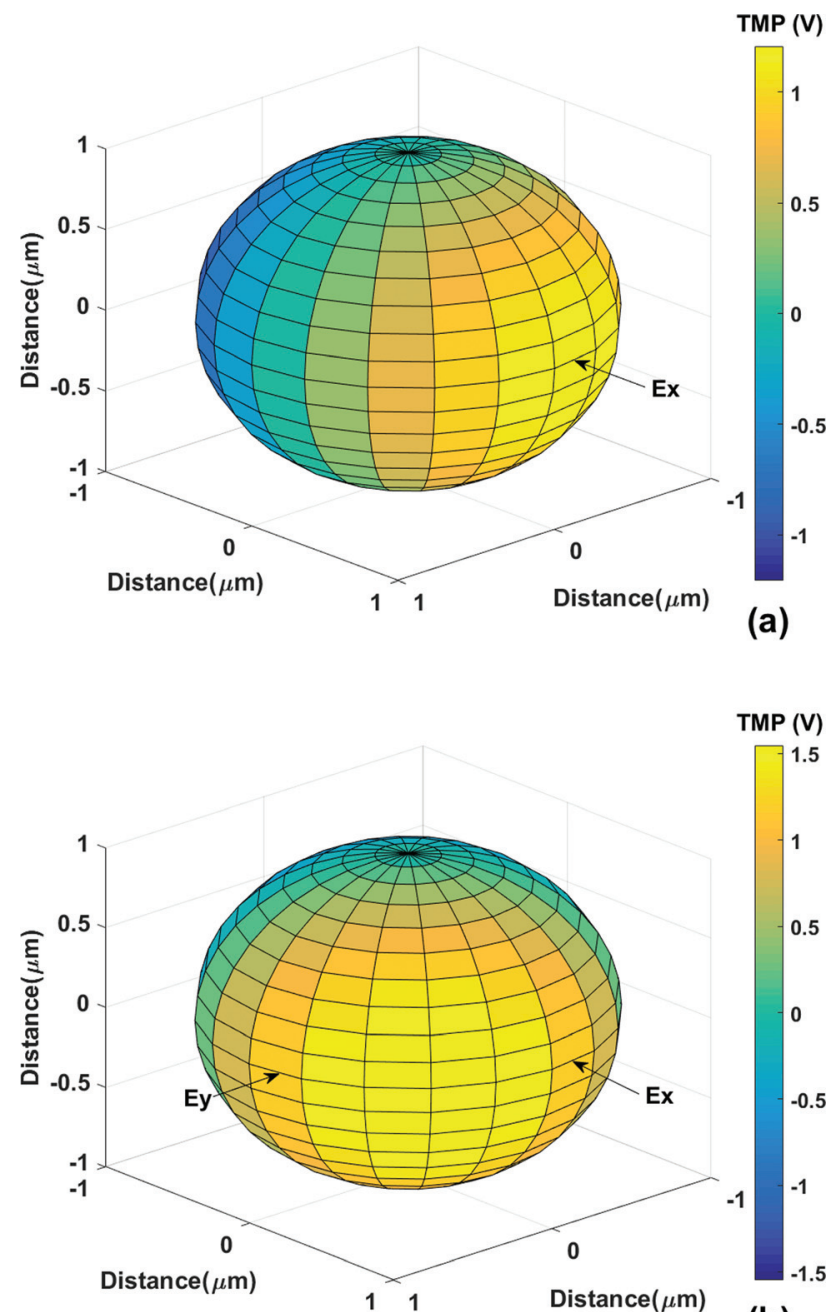

(b)

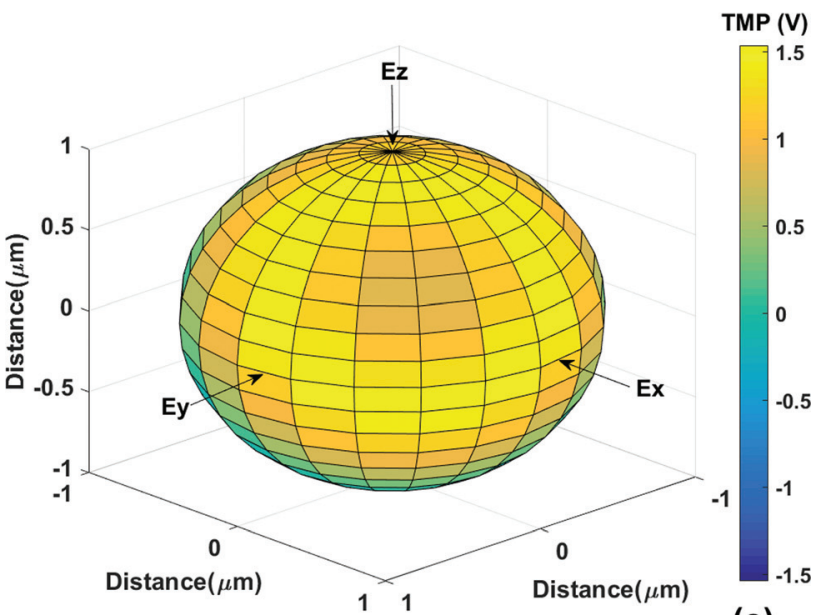

(c)

FIG. 10. Transmembrane potentials on the cell membrane surface for the pulse train of Fig. 8 at the $30 \mathrm{~ns}$ time instant. (a) Pulses applied only along the $\mathrm{x}$-axis, (b) pulses applied along both the $\mathrm{x}$ - and $\mathrm{y}$-directions, and (c) pulses applied along all three $\mathrm{x}-, \mathrm{y}-$, and $\mathrm{z}$-directions simultaneously. 
Since the pulses in the train had a 40-ns duration, with 1.5-ns rise-, 1.5-ns fall-times, and 60-ns off times, the field at $30 \mathrm{~ns}$ was $80 \mathrm{kV} / \mathrm{cm}$. In Fig. 10(a), the monopolar pulses were applied with the electric fields along the $\mathrm{x}$-axis. For Fig. 9(b), the external electric fields were along both the $\mathrm{x}$ - and $\mathrm{y}-$ directions; while Fig. 10(c) shows the results obtained when pulse trains were simultaneously applied along all three axes. In Fig. 10(a), the green and bluish-green regions in the $\mathrm{y}-\mathrm{z}$ plane have relatively low TMP values since this area is normal to the applied x-direction. The polar caps along the $\mathrm{X}$-axis are the regions that exhibit high TMP values exceeding a $1 \mathrm{~V}$ magnitude (yellow color at the front end, dark blue towards the back). The result of Fig. 10(b) shows a much larger fraction of the surface area with higher TMP magnitudes, while Fig. 10(c) reveals the highest percentage of the membrane having TMP magnitudes near, or in excess of, $1 \mathrm{~V}$ which is a convenient benchmark for electroporation. Since pore density scales nonlinearly with TMP magnitudes, a significantly high number of pores are predicted upon the simultaneous application of pulses in the three orthogonal directions. Though pore density distribution over the membrane surface could have been shown, the TMP profile was chosen here because of its simpler, narrow range of values. For completeness, the fractional values for the electroporated membrane area obtained from the simulations were $34 \%$, $36 \%$, and $63.95 \%$ for external fields applied along the $\mathrm{x}-\mathrm{x}$ and $\mathrm{y}^{-}$, and $\mathrm{x}-, \mathrm{y}-$ and $\mathrm{z}$-directions, respectively.

\section{CONCLUSIONS}

Electroporation is a useful technique for cellular biomanipulation, drug and gene delivery and for targeted apoptosis. An important practical consideration is the ionic throughput and delivery into cells (and tissues) that could be optimized by tailoring the electrical pulsing parameters. This contribution was a step in this direction, and presented a full three-dimensional, time-dependent analysis of ion inflows following external pulse application. The present simulations included both electrophoretic and diffusive transport, and comparisons were made between monopolar and bipolar pulse responses. In addition, the response driven by voltages applied at multiple electrodes was also probed.

It was shown that monopolar pulsing always led to higher and stronger cellular uptake. This prediction is in agreement with previous experimental reports and observations. ${ }^{57-59}$ For instance, in the experiments by Gianulis et al., ${ }^{57}$ it was shown that the electric field polarity reversal hinders the electroporative efficiency. They noted that the rate and amount of uptake for the marker dye YO-PRO-1 was consistently two-to-three fold higher for unipolar pulses as compared to the bipolar treatments. A similar conclusion was reached by Roth et al. ${ }^{58}$ and Sano and coworkers. ${ }^{59}$ It was also demonstrated through quantitative predictions that from a practical standpoint, multi-pronged electrode configurations would influence the degree of cellular uptake. Judicious pulse sequencing would then add to the overall benefits. Finally, it is possible that the creation of pressure transients $^{58}$ could add to the overall bio-response, or that temperature gradients set up due to such electric pulse application $^{60}$ could lead to even greater advantages. The latter will be analyzed and discussed elsewhere.

For completeness, we touch upon the aspect of diffusive cellular uptake over long scales, as reported in the experimental literature. ${ }^{37,38}$ Diffusion is certainly the logical process enabling transport over long time scales when the external driving fields have become zero. However, in the current model analyses, we have deliberately chosen to focus on the short time scales for the following reason. Transport at the long time scales will be guided in large part by the ability of pores to stay open and on details of the pore resealing mechanism and dynamics. The latter is a complicated problem and is not well understood at present. The simplest solution, especially for matching experimental data, might be to use various "fitting" time constants for pore closing. However, the physics is complicated. For example, the resealing times depend on the local membrane stresses which would not only be dependent on the pore radius, but also be dictated by the distribution of pores in the neighborhood. It is also controlled by the evolution of the elastic parameters such as bending moduli. ${ }^{61}$ Furthermore, over time, the stresses would change in a dynamic fashion. Finally, porepore coupling effects, especially in the context of highintensity pulsing which gives rise to a high density of nanopores (i.e., supraporation) as first suggested by Gowrishankar and Weaver, ${ }^{62}$ would complicate the problem even further. We have already shown that electroporation is a twofold process. ${ }^{63}$ The electric driving force produces a Maxwell stress at the membrane and works to rearrange lipid dipoles, cause openings and nanopores. However, water entry which is essential for useful transport, is facilitated by electrowetting, which is an important component of the overall process. The molecular system can thus function as an electrically driven hydrophobic toggle switch that changes the water permeability. The main issue though is that the pore closing times and their dynamics are not well understood, nor been well characterized. However, despite the greater accuracy and strong physics-based framework, the kinetics of pore closure observed in MD has so far not predicted pores being open for seconds or longer after a pulse application as seen in some experimental studies. ${ }^{64,65}$ Hence, the simple-minded concept of using some effective pore-closing times for fitting transport over long times, stands on shaky grounds. For this reason, we have chosen not to venture into this regime or simulate diffusion dominated behaviors over long time scales.

\section{ACKNOWLEDGMENTS}

Useful discussions with K. Schoenbach (ODU) are gratefully acknowledged.

\section{APPENDIX: AREA AND CURRENT CALCULATIONS}

The current continuity equation applied at each elemental volume shown in Fig. 1 leads to the following equation, which takes into account both conduction and displacement currents. The algebraic sum of the currents across each of the six surfaces on the elemental cube is taken into account: 


$$
\begin{aligned}
\sigma_{1} & \frac{V_{i-1, j, k}^{t}-V_{i, j, k}^{t}}{\Delta r} A_{1}+\sigma_{2} \frac{V_{i+1, j}^{t}-V_{i, j}^{t}}{\Delta r} A_{2}+\sigma_{3} \frac{V_{i, j-1, k}^{t}-V_{i, j, k}^{t}}{r \Delta \theta} A_{3}+\sigma_{4} \frac{V_{i, j+1, k}^{t}-V_{i, j, k}^{t}}{r \Delta \theta} A_{4} \\
+ & \left.\sigma_{5} \frac{V_{i, j, k-1}^{t}-V_{i, j, k}^{t}}{r \sin \theta \Delta \phi} A_{5}+\sigma_{6} \frac{V_{i, j, k+1}^{t}-V_{i, j, k}^{t}}{r \sin \theta \Delta \phi} A_{6}+\frac{\varepsilon_{1}\left[\frac{V_{i-1, j, k}^{t+1}-V_{i, j, k}^{t+1}}{\Delta t}-\frac{V_{i-1, j, k}^{t}-V_{i, j, k}^{t}}{\Delta r}\right] A_{1}}{\Delta r}\right] A_{2}+\frac{\varepsilon_{3}}{\Delta t}\left[\frac{V_{i, j-1, k}^{t+1}-V_{i, j, k}^{t+1}}{r \Delta \theta}-\frac{\left.V_{i, j-1, k}^{t}-V_{i, j, k}^{t}\right]}{r \Delta \theta}\right] A_{3} \\
+ & \frac{\varepsilon_{2}}{\Delta t}\left[\frac{V_{i+1, j, k}^{t+1}-V_{i, j, k}^{t+1}}{\Delta r}-\frac{V_{i+1, j, k}^{t}-V_{i, j, k}^{t}}{\Delta r}\right] A_{4} \\
& \left.+\frac{\varepsilon_{4}}{\Delta t}\left[\frac{V_{i, j+1, k}^{t+1}-V_{i, j, k}^{t+1}}{r \Delta \theta}-\frac{V_{i, j+1, k}^{t}-V_{i, j, k}^{t}}{r \Delta \theta}\right] A_{i, j}^{t}-\frac{V_{i, j, k-1}^{t}-V_{i, j, k}^{t}}{r \sin \theta \Delta \phi}\right] A_{5}+\frac{\varepsilon_{6}}{\Delta t}\left[\frac{V_{i, j, k+1}^{t+1}-V_{i, j, k}^{t+1}}{r \sin \theta \Delta \phi}-\frac{V_{i, j, k+1}^{t}-V_{i, j, k}^{t}}{r \sin \theta \Delta \phi}\right] A_{6}=0 .
\end{aligned}
$$

In the above, the areas $A_{i}$ are given as

$$
\begin{aligned}
& A_{1}=\left(r+\frac{\Delta r}{2}\right)^{2}\left[\cos \left(\theta-\frac{\Delta \theta}{2}\right)-\cos \left(\theta+\frac{\Delta \theta}{2}\right)\right] \Delta \varphi, \\
& A_{2}=\left(r-\frac{\Delta r}{2}\right)^{2}\left[\cos \left(\theta-\frac{\Delta \theta}{2}\right)-\cos \left(\theta+\frac{\Delta \theta}{2}\right)\right] \Delta \varphi, \\
& A_{3}=r \Delta r \sin \left(\theta-\frac{\Delta \theta}{2}\right) \Delta \varphi, \\
& A_{4}=r \Delta r \sin \left(\theta+\frac{\Delta \theta}{2}\right) \Delta \varphi, \\
& A_{5}=A_{6}=r \Delta r \Delta \theta \\
& A_{6}=r \Delta r \Delta \theta .
\end{aligned}
$$

Nodes with $j=0$ and $j=m$ have to be treated carefully since these grid points are independent of $k$. Only $I_{1}, I_{2}$, and $I_{4}$ are nonzero in the equation, and the sum of $\mathrm{I}_{5}$ and $\mathrm{I}_{6}$ would be zero. For $j=0$ and $1<i<N_{i}$, Eq. (A1) effectively becomes

$$
\begin{aligned}
N_{k} & \sigma_{1} \frac{V_{i-1, j}^{t}-V_{i, j}^{t}}{\Delta r} A_{1}+n \sigma_{2} \frac{V_{i+1, j}^{t}-V_{i, j}^{t}}{\Delta r} A_{2} \\
& +\sum_{k=0}^{N_{k}} \sigma_{4} \frac{V_{i, j+1, k}^{t}-V_{i, j}^{t}}{r \Delta \theta} A_{4} \\
+ & \frac{\varepsilon_{1}}{\Delta t}\left[\frac{V_{i-1, j}^{t+1}-V_{i, j}^{t+1}}{\Delta r}-\frac{V_{i-1, j}^{t}-V_{i, j}^{t}}{\Delta r}\right] A_{1} \\
+ & \frac{\varepsilon_{2}}{\Delta t}\left[\frac{V_{i+1, j}^{t+1}-V_{i, j}^{t+1}}{\Delta r}-\frac{V_{i+1, j}^{t}-V_{i, j}^{t}}{\Delta r}\right] A_{2} \\
+ & \sum_{k=0}^{N_{k}} \frac{\varepsilon_{4}}{\Delta t}\left[\frac{V_{i, j+1, k}^{t+1}-V_{i, j}^{t+1}}{r \Delta \theta}-\frac{V_{i, j+1 . k}^{t}-V_{i, j}^{t}}{r \Delta \theta}\right] A_{4}=0 .
\end{aligned}
$$

And for $j=N_{j}$ and $l<i<N_{i}$, Eq. (A1) effectively becomes

$$
\begin{aligned}
& N_{k} \sigma_{1} \frac{V_{i-1, j}^{t}-V_{i, j}^{t}}{\Delta r} A_{1}+n \sigma_{2} \frac{V_{i+1, j}^{t}-V_{i, j}^{t}}{\Delta r} A_{2} \\
& +\sum_{k=0}^{N_{k}} \sigma_{4} \frac{V_{i, j-1, k}^{t}-V_{i, j}^{t}}{r \Delta \theta} A_{4}+\frac{\varepsilon_{1}}{\Delta t}\left[\frac{V_{i-1, j}^{t+1}-V_{i, j}^{t+1}}{\Delta r}-\frac{V_{i-1, j}^{t}-V_{i, j}^{t}}{\Delta r}\right] A_{1} \\
& +\frac{\varepsilon_{2}}{\Delta t}\left[\frac{V_{i+1, j}^{t+1}-V_{i, j}^{t+1}}{\Delta r}-\frac{V_{i+1, j}^{t}-V_{i, j}^{t}}{\Delta r}\right] A_{2} \\
& +\sum_{k=0}^{N_{k}} \frac{\varepsilon_{4}}{\Delta t}\left[\frac{V_{i, j-1, k}^{t+1}-V_{i, j}^{t+1}}{r \Delta \theta}-\frac{V_{i, j-1 . k}^{t}-V_{i, j}^{t}}{r \Delta \theta}\right] A_{4}=0 .
\end{aligned}
$$

${ }^{1}$ E. Neumann and K. Rosenheck, J. Membr. Biol. 10, 279 (1972).

${ }^{2}$ R. Stampfli, An. Acad. Bras. Cienc. 30, 57 (1958).

${ }^{3}$ T. Y. Tsong, Biophys. J. 60, 297 (1991).

${ }^{4}$ J. C. Weaver and Y. A. Chizmadzhev, Bioelectrochem. Bioenerg. 41, 135 (1996).

${ }^{5}$ I. G. Abidor, V. B. Arakelian, Y. Chemomordik, A. Chizmadzhev, V. F. Pastushenko, and M. R. Tarasevich, Bioelectrochem. Bioenerg. 6, 37 (1979). ${ }^{6} \mathrm{~S}$. H. Meglic and T. Kotnick, "Electroporation-based applications in biotechnology," in Handbook of Electroporation, edited by D. Miclavcic (Springer International Publishing, Cham, Switzerland, 2016).

${ }^{7}$ R. P. Joshi and K. H. Schoenbach, Crit. Rev. Bio-Med. Eng. 38, 255 (2010).

${ }^{8}$ M. P. Rols and J. Teissie, Biophys. J. 65, 409 (1998).

${ }^{9}$ K. H. Schoenbach, R. P. Joshi, J. Kolb, N. Chen, M. Stacey, P. Blackmore, E. S. Buescher, and S. J. Beebe, Proc. IEEE 92, 1122 (2004).

${ }^{10}$ R. Nuccitelli, J. C. Berridge, Z. Mallon, M. Kreis, B. Athos, and P. Nuccitelli, "Nanoelectroablation of murine tumors triggers a CD8dependent inhibition of secondary tumor growth," PLoS One 10, e0134364 (2015).

${ }^{11}$ T. B. Napotnik, M. Reberšek, P. T. Vernier, B. Mali, and D. Miklavčič, Bioelectrochemistry 110, 1 (2016).

${ }^{12}$ S. J. Beebe, P. F. Blackmore, J. White, R. P. Joshi, and K. H. Schoenbach, Physiol. Meas. 25, 1077 (2004).

${ }^{13}$ P. T. Vernier, Y. Sun, L. Marcu, S. Salemi, C. M. Craft, and M. A. Gundersen, Biochem. Biophys. Res. Commun. 310, 286 (2003).

${ }^{14}$ R. Nuccitelli, U. Pliquett, X. Chen, W. Ford, R. J. Swanson, S. J. Beebe, J. F. Kolb, and K. H. Schoenbach, Biochem. Biophys. Res. Commun. 343, 351 (2006).

${ }^{15}$ R. Nuccitelli, R. Wood, M. Kreis, B. Athos, J. Huynh, K. Liu, P. Nuccitelli, and E. H. Epstein, Exp. Dermatol. 23, 135 (2014).

${ }^{16}$ R. P. Joshi, A. Mishra, J. Song, A. Pakhomov, and K. H. Schoenbach, IEEE Trans. Biomed. Eng. 55, 1391 (2008).

${ }^{17}$ K. H. Schoenbach, B. Hargrave, R. P. Joshi, J. F. Kolb, R. Nuccitelli, C. Osgood, A. Pakhomov, M. Stacey, R. J. Swanson, J. White, S. Xiao, J. Zhang, S. J. Beebe, P. F. Blackmore, and E. S. Buescher, IEEE Trans. Dielectr. Electr. Insul. 14, 1088 (2007).

${ }^{18}$ N. Jourabchi, K. Beroukhim, B. A. Tafti, S. T. Kee, and E. W. Lee, Gastrointest. Intervention 3, 8 (2014).

${ }^{19}$ M. Ahmed, C. L. Brace, F. T. Lee, Jr., and S. N. Goldberg, Radiology 258, 351 (2011).

${ }^{20}$ D. Miklavčič, B. Mali, B. Kos, R. Heller, and G. Serša, Biomed. Eng. Online 13, 29 (2014).

${ }^{21}$ G. Schmidt, I. Juhasz-Böss, E.-F. Solomayer, and D. Herr, Geburtshilfe Frauenheilkd. 74, 557 (2014); G. Pucihar, T. Kotnik, M. Kandušer, and D. Miklavčič, Bioelectrochemistry 54, 107 (2001).

${ }^{22}$ G. L. Prasanna and T. Panda, Bioprocess Eng. 16, 261 (1997).

${ }^{23}$ K. H. Schoenbach, S. J. Beebe, and E. S. Buescher, Bioelectromagnetics 22, 440 (2001).

${ }^{24}$ J. Teissie, N. Eynard, B. Gabriel, and M. P. Rols, Adv. Drug Delivery Rev. 35, 3 (1999).

${ }^{25}$ T. S. Santra, P.-C. Wang, and F. G. Tseng, "Electroporation based drug delivery and its applications," in Advances in Micro/Nano Electromechanical Systems and Fabrication Technologies, edited by K. Takahata (InTech Publishers, London, UK, 2013).

${ }^{26}$ K. Kinosita, Jr., I. Ashikawa, N. Saita, H. Yoshimura, H. Itoh, K. Nagayama, and A. Ikegami, Biophys. J. 53, 1015 (1988). 
${ }^{27}$ M. Hibino, H. Itoh, and K. Kinosita, Jr., Biophys. J. 64, 1789 (1993).

${ }^{28}$ G. Pucihar, T. Kotnik, D. Miklavčič, and J. Teissié, Biophys. J. 95, 2837 (2008).

${ }^{29}$ M. M. Sadik, M. Yu, M. Zheng, J. D. Zahn, J. W. Shan, D. I. Shreiber, and H. Lin, Biophys. J. 106, 801 (2014).

${ }^{30}$ K. H. Schoenbach, A. G. Pakhomov, I. Semenov, S. Xiao, O. N. Pakhomova, and B. L. Ibey, Bioelectrochemistry 103, 44 (2015).

${ }^{31}$ R. A. Gilbert, M. J. Jaroszeski, and R. Heller, Biochim. Biophys. 1334, 9 (1997).

${ }^{32}$ H. Garner, G. A. Hofmann, S. B. Dev, and G. S. Nanda, IEEE Eng. Med. Biol. Mag. 15, 124 (1996).

${ }^{33}$ H. Garner, E. Tekle, R. D. Astumian, and P. B. Chock, Proc. Natl. Acad. Sci. U.S.A. 88, 4230 (1991).

${ }^{34}$ J. Teissie and M. P. Rols, Biophys. J. 65, 409 (1993).

${ }^{35}$ T. Kotnik, L. M. Mir, K. Flisar, M. Puc, and D. Miklavcic, Bioelectrochemistry 54, 83 (2001).

${ }^{36}$ T. Kotnik, G. Pucihar, M. Rebersek, D. Miklavcic, and L. M. Mir, "Role of pulse shape in cell membrane electropermeabilization," Biochim. Biophys. Acta 1614, 193-200 (2003).

${ }^{37}$ C. Faurie, M. Rebersek, M. Golzio, M. Kanduser, J.-M. Escoffre, M. Pavlin, J. Teissie, D. Miklavcic, and M.-P. Rols, "Electro-mediated gene transfer and expression are controlled by the life-time of DNA/membrane complex formation," J. Gene Med. 12, 117-125 (2010).

${ }^{38}$ B. L. Ibey, J. C. Ullery, O. N. Pakhomova, C. C. Roth, I. Semenov, H. T. Beier, M. Tarango, S. Xiao, K. H. Schoenbach, and A. G. Pakhomov, Biochem. Biophys. Res. Commun. 443, 568 (2014).

${ }^{39}$ A. G. Pakhomov, I. Semenov, S. Xiao, O. N. Pakhomova, B. Gregory, K. H. Schoenbach, J. C. Ullery, H. T. Beier, S. R. Rajulapati, and B. L. Ibey, Cell. Mol. Life Sci. 71, 4431 (2014).

${ }^{40}$ I. Daskalov, N. Mudrov, and E. Peycheva, IEEE Eng. Med. Biol. Mag. 18, 62 (1999).

${ }^{41}$ M. B. Sano, C. B. Arena, K. R. Bittleman, M. R. DeWitt, H. J. Cho, C. S. Szot, D. Saur, J. M. Cissel, J. Robertson, Y. W. Lee, and R. V. Davalos, Sci. Rep. 5, 14999 (2015).

${ }^{42}$ D. Miklavcic, K. Beravs, D. Semrov, M. Cemazar, F. Demsar, and G. Sersa, Biophys. J. 74, 2152 (1998).

${ }^{43}$ K. Brandinsky and I. Daskalov, Bioelectrochem. Bioenerg. 48, 201 (1999).

${ }^{44}$ R. P. Joshi and Q. Hu, IEEE Trans. Biomed. Eng. 58, 2860 (2011).
${ }^{45}$ Q. Hu and R. P. Joshi, IEEE Trans. Biomed. Eng. 56, 1617 (2009).

${ }^{46}$ S. B. Dev, D. Dhar, and W. Krassowska, IEEE Trans. Biomed. Eng. 50, 1296 (2003).

${ }^{47}$ R. P. Joshi and K. H. Schoenbach, "Electric fields in biological cells and membranes," in Electromagnetic Fields in Biological Systems (CRC Press, New York), pp. 71-114.

${ }^{48}$ R. P. Joshi, Q. Hu, and K. H. Schoenbach, IEEE Trans. Plasma Sci. 32, 1677 (2004).

${ }^{49}$ J. C. Neu and W. Krassowska, Phys. Rev. E 59, 3471 (1999).

${ }^{50}$ A. Barnett and J. C. Weaver, Bioelectrochem. Bioenerg. 25, 163 (1991).

${ }^{51}$ V. F. Pastushenko and Yu. A. Chizmadzhev, Biofizika 27, 475 (1982).

${ }^{52}$ S. A. Freeman, M. A. Wang, and J. C. Weaver, Biophys. J. 67, 42 (1994).

${ }^{53}$ R. W. Glaser, S. L. Leikin, L. V. Chernomordik, V. F. Pastushenko, and A. I. Sokirko, Biochim. Biophys. Acta 940, 275 (1988).

${ }^{54}$ R. P. Joshi, Q. Hu, R. Aly, K. H. Schoenbach, and H. P. Hjalmarson, Phys. Rev. E 64, 11913 (2001).

${ }^{55}$ J. C. Weaver, personal communication (2016).

${ }^{56}$ W. Frey, J. A. White, R. O. Price, P. F. Blackmore, R. P. Joshi, R. Nuccitelli, S. J. Beebe, K. H. Schoenbach, and J. Kolb, Biophys. J. 90, 3608 (2006).

${ }^{57}$ E. C. Gianulis, J. Lee, C. Jiang, S. Xiao, B. L. Ibey, and A. G. Pakhomov, Sci. Rep. 5, 13818 (2015)

${ }^{58}$ C. C. Roth, R. A. Barnes, Jr., B. L. Ibey, H. T. Beier, L. C. Mimun, S. M. Maswadi, M. Shadaram, and R. D. Glickman, Sci. Rep. 5, 15063 (2015).

${ }^{59}$ M. B. Sano, C. B. Arena, M. R. DeWitt, D. Saur, and R. V. Davalos, Bioelectrochemistry 100, 69 (2014).

${ }^{60}$ J. Song, A. L. Garner, and R. P. Joshi, Phys. Rev. Appl. 7, 024003 (2017).

${ }^{61}$ R. P. Joshi and Q. Hu, Med. Biol. Eng. Comput. 48, 837 (2010).

${ }^{62}$ T. R. Gowrishankar and J. C. Weaver, Biochem. Biophys. Res. Commun. 349, 643 (2006).

${ }^{63}$ Q. Hu, Z. Zhang, H. Qiu, M. G. Kong, and R. P. Joshi, Phys. Rev. E 87, 032704 (2013).

${ }^{64}$ Z. A. Levine and P. T. Vernier, J. Membr. Biol. 236, 27 (2010).

${ }^{65}$ W. F. Bennett, N. Sapay, and D. P. Tieleman, Biophys J. 106, 210 (2014). 